\title{
Optimum Power Selection Algorithms in Aloha Networks: Random and Deterministic Approaches
}

\author{
Behrouz Khoshnevis and Babak H. Khalaj
}

\begin{abstract}
In this paper, we discuss the optimum random and deterministic power selection algorithms in Aloha networks, where nodes run Exponential Backoff (EB) for contention resolution. In random case, the transmission power of a packet is selected from the available power levels, based on a predetermined probability mass function, while with the deterministic algorithms the transmission power of a packet is a deterministic function of the number of collisions the packet has experienced. Most of the related works in the literature have not addressed the power-throughput characteristics of the power selection algorithms for use in practical system designs and, therefore, this subject has been the major motivation of this paper. For the random case, we will derive optimum random power selection algorithms for unconstrained and constrained power budget scenarios and the corresponding optimum power-throughput characteristics will be presented for the latter case based on perfect capture model. Next, we will introduce a method to extend these results to SIR-based capture model, which will result in suboptimum power steps and the sub-optimum power-throughput characteristics for the random case. This characteristic will reveal power budget requirements for the target throughput values.

In the next step, deterministic power selection algorithms will be introduced and discussed with unconstrained and constrained power budget scenarios and sub-optimum power-throughput characteristic will be derived based on the perfect model. Similar methods, introduced for the random case, may be used to adapt the results to SIR-based model. Finally, by comparing the presented power-throughput characteristics, it will be demonstrated that optimum random and sub-optimum deterministic power selection algorithms have very similar behaviors. Therefore, noting that deterministic algorithms do not require any random power generator block, they may be preferred to random algorithms in similar scenarios.
\end{abstract}

Index Terms-Aloha, Exponential Backoff, throughput, power budget, capture effect.

\section{INTRODUCTION}

I $\mathrm{T}$ is well established that throughput of Aloha networks can be improved by using multiple power levels at transmitters, which is known as the power differentiation technique. Consider a network of nodes which are trying to send their packets to a common destination. With multiple power levels at transmitters, there is a chance that a packet with high enough transmission power will capture the channel, even if it has experienced collision with some other simultaneously transmitted packets. This phenomenon, known as "capture effect", has been studied based on two major models. The perfect model assumes that among colliding packets, one

During this research B. Khoshnevis has been with the Advanced Communication Research Institute, Sharif University of Technology, Tehran, Iran. Email: b_khoshnevis@alum.sharif.edu .

B. H. Khalaj is with the Electrical Engineering faculty of Sharif University of Technology, Tehran, Iran. Email: khalaj@sharif.edu . with the maximum power will capture the channel. This assumption, although unrealistic, simplifies the analysis. With the SIR-based model, a packet may capture the channel if its transmission power is at least $S I R_{\min }$ times greater than the interference caused by other simultaneously transmitted packets. $S I R_{\min }$, also known as capture ratio, is the minimum signal-to-interference ratio required for a valid reception at the central receiver and its value depends on the modulation and coding schemes used.

Throughput improvement algorithms which exploit capture effect have been widely studied in the literature. In [1], nodes have been partitioned into disjoint sets, where the nodes of each set transmit their packets with a predetermined power level. Although the overall throughput of the network will increase with such scheme, the algorithm acts more in favor of nodes with larger powers. This shortcoming has motivated the study of random power selection algorithms, in which, nodes select their transmission powers based on a power probability mass function (PMF). In [2], the author has assumed uniform PMF for power selection and the throughputdelay characteristic of network has been studied based on a specific capture model with the assumption of linearly equispaced power levels. The authors of [3] have done a comprehensive work in formulating the throughput optimization problem both with perfect and SIR-based capture models, in order to derive optimum PMFs and power levels. One of the major conclusions of their work is that linearly equi-spaced power levels are too far from optimum power levels and logarithmically equi-spaced power levels can be considered as sub-optimum solution to their optimization problem. In [4], the power budget has been taken into account in the optimization problem where the author has optimized the power selection PMF according to a power budget constraint. A search method has been presented to derive optimum power levels and PMFs, however, this work lacks presentation of a general power-throughput characteristic, which has been one of the major motivations of our paper. Below, we list some common features of the aforementioned works and describe how our approach is related to them.

i) The aforementioned works have not considered power selection algorithms with any specific retransmission scheme and their results are specifically dependent on the number of nodes in the network or the offered load, both of which are typically unknown variables in Aloha networks. Our approach, on the other hand, has been based on an exponential backoff retransmission scheme model. With the presented modeling and also by assuming infinite number of nodes, our analysis becomes independent of the offered load and the number of 
nodes. As it will be shown, the analysis results will also be applicable to networks with large enough finite number of nodes. Moreover, the assumption of a backoff algorithm which counts the number of collisions, enables us to introduce deterministic power selection algorithms, which have not been mentioned in earlier literature. Using these algorithms, the transmission power of a packet will be a deterministic function of the number of collisions and, therefore, there will be no need for a random power generator block.

ii) Although the average power (per successfully transmitted packet) is clearly the main constraint of power differentiation techniques, these works have not provided a general power-throughput characteristic to be used in the design process. We address this problem as we consider random and deterministic power selection algorithms for the constrained power budget scenarios. The output of this analysis will be the optimum random and sub-optimum deterministic powerthroughput characteristic, which will be derived based on perfect capture model.

iii) Because of the high complexity of SIR-based model, earlier results are mainly based on numerical methods and analytical approaches are only provided for the perfect model scenarios. Our approach toward the problem has been mainly analytical and in spite of using perfect model for our calculations, we will introduce an approximate method to adapt the perfect model results to SIR-based capture model. The output of this approximation for the random case will be sub-optimum power-throughput characteristic and sub-optimum power steps, number of power levels, PMFs and backoff factors for the given power budget constraints. The presented approach can also be used for deterministic algorithms in order to adapt the presented perfect model results to SIR-based model. The accuracy of the approximate analysis will be verified through simulation results.

It should be mentioned that, in addition to the aforementioned works, some recent works have been done on power differentiation techniques, which will not be discussed here in order to preserve the coherence of earlier references. Interested readers are referred to [5]-[11] and the references therein.

Before describing the organization of the paper, we will list the major assumptions of our analysis. The following assumptions have been made for ease of analysis and are globally accepted in this paper, unless explicitly mentioned.

- Infinite number of nodes send their packets to a common destination through a slotted channel and every node gets informed of its packet's status (success or collision) at the end of the transmission slot, using some implicit acknowledging method. The nodes exploit exponential backoff retransmission scheme for contention resolution.

- Saturation condition has been assumed, where the packet queues of the nodes never get empty. The saturation model of exponential backoff will be used for our analysis.

- Path-loss and fading characteristics of the radio channel have been ignored. Therefore, only the transmission power of the packets have been considered in the analysis of capture effect. In fact, this assumption may be replaced by a weaker one; the nodes have almost equal distances from the central receiver and the path-loss characteristics are the same for all transmission pairs.

The outline of the paper is as follows. First, we will discuss the random algorithms. In section II, the Exponential Backoff (EB) model with random power selection algorithms will be discussed and the basic relations will be presented. In section III, we will derive Optimum Random power Selection Algorithms (ORPSAs) for the unconstrained budget scenario. The constrained power budget scenario, perfect model optimum power-throughput characteristics and the adaptation of the results to the SIR-based model will be discussed in IV.

Next, we will go on to the deterministic algorithms. Section $\mathrm{V}$ will present EB model with deterministic power selection algorithms and the basic relations for this scenario will be derived. In section VI, unconstrained power budget scenario will be discussed and it will be shown that the throughput of ORPSA can be considered as an upper bound for the throughput of Optimum Deterministic Power Selection Algorithm (ODPSA). Subsequently, we will introduce a search method for ODPSA, based on which, sub-optimum algorithms (subODPSAs) will be presented. The power-constrained scenario will be discussed in section IV and the corresponding perfect model sub-optimum power-throughput characteristic will be derived and it will be shown to be very close to the ORPSA characteristic.

\section{MODELING OF EXPONENTIAL BACKOFF WITH RANDOM POWER SELECTION ALGORITHMS}

The saturation condition, in which the nodes have always some packets pending transmission, has been discussed in [12]. We have adopted a simple modeling of EB under saturation condition, presented in [13], in order to describe the random power selection scenario. With this model, a node in state $n \geq 0$, transmits its packet with randomly chosen power and faces collision with probability of $p_{c}$, which leads it to state $n+1$, otherwise the node goes back to state 0 (Fig. $1)$. The contention window size at state $n$ is $W_{n}=W_{0} r^{n}$, where $W_{0}$ is the initial contention window size and $r$ is the backoff factor. The transmission power is selected from the set of $L$ available power levels, $\left\{P_{0}, P_{1}, \ldots, P_{L-1}\right\}$, according to the PMF, $\zeta=\left\{\zeta_{0}, \zeta_{1}, \ldots, \zeta_{L-1}\right\}$. Note that, $P_{i+1}>P_{i}$, for $0 \leq i<\bar{L}-1$. Also, $\zeta_{i}>0$, for $0 \leq i \leq L-1$, and $\sum_{i=0}^{L-1} \zeta_{i}=1$.

The following theorem summarizes the basic relations we will use in analysis of random power selection algorithms. It should be noted that the perfect capture model has been used for calculating the probability of collision.

Theorem 1: Consider a network of $N$ nodes which use the EB model of Fig. 1 for contention resolution and power selection. By defining $p_{t}, p_{c}, p_{s u c c}$ and $P_{a v}$ as the average probability of transmission, collision ${ }^{1}$, successful transmission $^{2}$ and average required power for a successful transmis-

\footnotetext{
${ }^{1}$ Hereafter, by "collision", the case of simultaneous transmissions is meant, where none of the packets has been able to capture the channel.

${ }^{2}$ We will use the term "throughput" for the average probability of successful transmission, since it represents the amount of channel utilization.
} 


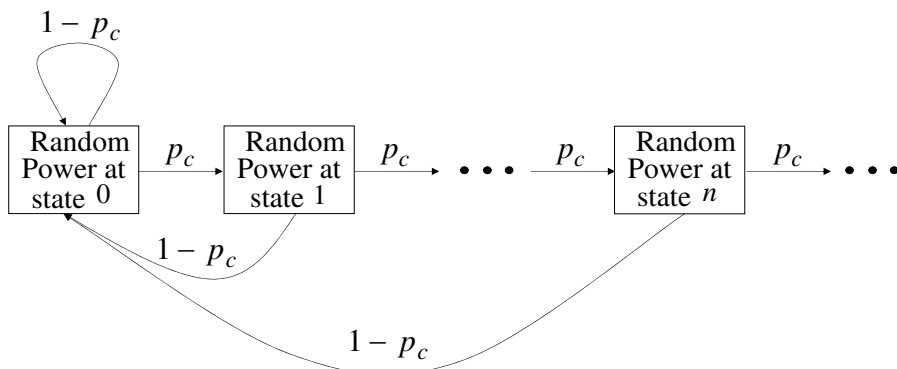

Fig. 1. EB model with random power selection algorithm. Since the same random algorithm selects the transmission power in all states, the probability of collision will in average be independent of the number of collisions.

sion, respectively, we will have:

$$
\begin{gathered}
p_{t}=\frac{2\left(1-r p_{c}\right)}{W_{0}\left(1-p_{c}\right)+1-r p_{c}} \\
1-p_{c}=\sum_{i=0}^{L-1} \zeta_{i}\left(1-p_{t} \sum_{j=i}^{L-1} \zeta_{j}\right)^{N-1} \\
p_{s u c c}=N p_{t}\left(1-p_{c}\right) \\
P_{a v}=\sum_{i=0}^{L-1} \zeta_{i} P_{i} /\left(1-p_{c}\right)
\end{gathered}
$$

Proof:

(1) has been proven in [13].

Based on the assumption of perfect model, a packet with power $P_{i}$ will capture the channel iff no other packet has been transmitted with power level equal to or greater than $P_{i}$. This proves (2).

$p_{\text {succ }}$ is the probability that a node out of $N$ nodes makes a transmission and does not face collision. This proves (3).

A sample power required for a successful transmission can be expressed as $P=\sum_{i=0}^{\infty} p_{c}{ }^{i}\left(1-p_{c}\right) \sum_{j=0}^{i} P_{i}^{(j)}$,where $P_{i}^{(j)}, 0 \leq j \leq i$, are consecutive i.i.d. power levels used for retransmitting a packet until a successful transmission occurs at the $i^{\text {th }}$ transmission. Defining $P_{a v}$ as $E\{P\}$ and noting that $E\left\{P_{i}^{(j)}\right\}=\sum_{k=0}^{L-1} \zeta_{k} P_{k}$, the proof of (4) will be complete. It should be noted that, our system model considers the average condition, where the average probability of collision has been assumed to be independent of transmission powers, $P_{i}^{(j)}$. In fact, both the number of retransmissions $i$, and probabilities of collision depend on $P_{i}^{(j)}$. Considering this dependence, calculation of $P_{a v}$ will be a little bit more complex, however, after some manipulations, similar to the approach presented in [4], the final result will be the same as (4).

For the case of infinite number of nodes, which is the main focus of this paper, we will have:

$$
\lim _{N \rightarrow \infty} p_{t}=0
$$

In order to prove (5), suppose that $\lim _{N \rightarrow \infty} p_{t}>0$, then from (2), we will have $p_{c}=1$, as $N$ tends to infinity, which is a contradiction. Also, by combining (5) and (1),

$$
\lim _{N \rightarrow \infty} p_{c}=1 / r .
$$

Statements similar to (5) and (6) have also been reported in [13]. Considering (2), (3) and (6), we will have the following equation for the case of infinite number of nodes ${ }^{3}$ :

$$
p_{s u c c}=N p_{t}(1-1 / r)=\sum_{i=0}^{L-1} N p_{t} \zeta_{i} e^{-N p_{t} \sum_{j=i}^{L-1} \zeta_{j}} .
$$

Based on the presented system model and relations, we will go through the optimization problem in sections III and IV.

\section{ORPSA WITH UNCONSTRAINED POWER BUdGet}

In this section, we will discuss the optimization problem without considering any limit on the average power. We will derive ORPSA both for fixed and variable backoff factors.

\section{A. Fixed Backoff Factor}

Our purpose is to find the optimum values of elements of $\zeta$, such that $p_{\text {succ }}=N p_{t}(1-1 / r)$ is maximized, while following conditions hold:

$$
\begin{gathered}
\sum_{i=0}^{L-1} \zeta_{i}=1 \\
\sum_{i=0}^{L-1} \zeta_{i} e^{-N p_{t} \sum_{j=i}^{L-1} \zeta_{j}}=1-1 / r
\end{gathered}
$$

By defining $\beta_{i}=N p_{t} \sum_{j=i}^{L-1} \zeta_{j}$ for $0 \leq i \leq L-1, \beta_{-1}=\infty$, $\beta_{L}=0$ and noting that $r$ is fixed, the problem can be rephrased as maximizing $\beta_{0}=N p_{t}$, while satisfying the following condition:

$$
\sum_{i=0}^{L-1}\left(\beta_{i}-\beta_{i+1}\right) e^{-\beta_{i}}=\beta_{0}(1-1 / r) .
$$

By differentiating with respect to $\beta_{1}, \beta_{2}, \ldots, \beta_{L-1}$ we will have:

$$
\beta_{i+1}-\beta_{i}=e^{\beta_{i}-\beta_{i-1}}-1,
$$

for $1 \leq i \leq L-1$. Substituting (9) in (8), we will arrive at the following system of equations for $i=1,2, \ldots, L-1$ :

$$
\begin{gathered}
\beta_{0}(1-1 / r)+\left(1-\beta_{0}+\beta_{1}\right) e^{-\beta_{0}}-e^{-\beta_{L-1}}=0, \\
\beta_{i+1}-\beta_{i}=e^{\beta_{i}-\beta_{i-1}}-1 .
\end{gathered}
$$

By solving these equations, $\beta_{i}$ can be calculated, based on which the optimum $\underline{\zeta}$ can be expressed as, $\zeta_{i}=$ $\left(\beta_{i}-\beta_{i+1}\right) / \beta_{0}$. It can be shown by induction that, $\beta_{i}>\beta_{i+1}$, for $0 \leq i \leq L-1$, which ensures that $\zeta_{i}>0$.

In order to prove the inefficiency of using fixed backoff factors with ORPSAs, we will calculate the throughput of the derived ORPSA with infinite number of power levels. By defining the sequence of functions, $g_{1}(x)=x$ and $g_{k+1}(x)=$ $1-e^{-g_{k}(x)}$ for $k \geq 0$, it should be obvious from (9) that, $\beta_{k}-$ $\beta_{k+1}=g_{k+1}\left(\beta_{0}-\beta_{1}\right)$ for $k=0,1, \ldots, L-1$. Consequently,

\footnotetext{
${ }^{3}$ Noting that most of the equations of this paper are related to the "infinite nodes" scenario, the $\lim _{N \rightarrow \infty}$ symbol will be omitted for convenience. Consideration of "finite nodes" case will be explicitly mentioned.
} 
we will have $\beta_{0}=\sum_{k=1}^{L} g_{k}\left(\beta_{0}-\beta_{1}\right)$, therefore noting that $\beta_{0}=N p_{t}$, throughput can be expressed as:

$$
\begin{gathered}
\lim _{L \rightarrow \infty} p_{\text {succ }}=\lim _{L \rightarrow \infty} \beta_{0}(1-1 / r) \\
=\left.(1-1 / r) \sum_{k=1}^{\infty} g_{k}(x)\right|_{x=\lim _{L \rightarrow \infty}\left(\beta_{0}-\beta_{1}\right) .}
\end{gathered}
$$

However, it can be shown that $\sum_{k=1}^{\infty} g_{k}(x)$ diverges for any $x>0$. Therefore, for a meaningful value of $p_{\text {succ }}$, $\lim _{L \rightarrow \infty}\left(\beta_{0}-\beta_{1}\right)$ needs to be zero. Also, the last term of the above summation needs to tend to zero as $L$ tends to infinity, therefore, $\lim _{L \rightarrow \infty} \beta_{L-1}=\lim _{L \rightarrow \infty} g_{L}\left(\beta_{0}-\beta_{1}\right)=$ 0 . Combining these with (10), we will have,

$$
\lim _{L \rightarrow \infty} \beta_{0}(1-1 / r)+e^{-\lim _{L \rightarrow \infty} \beta_{0}}-1=0
$$

or,

$$
\lim _{L \rightarrow \infty} p_{\text {succ }}+e^{-\lim _{L \rightarrow \infty} p_{\text {succ }} /(1-1 / r)}-1=0 .
$$

It is obvious from (11) that, for any $r>1, \lim _{L \rightarrow \infty} p_{\text {succ }}<1$. This observation proves the inefficiency of fixed backoff factor scenario and encourages the use of backoff factors which depend on the number of power levels. We will discuss this problem in the next subsection.

\section{B. Variable Backoff Factor}

In this subsection, we will derive ORPSA with EB retransmission scheme, for which the backoff factor depends on the number of power levels. The problem is to find the optimum $\zeta$, such that $p_{\text {succ }}$ is maximized, while $\sum_{i=0}^{L-1} \zeta_{i}=1$. Using the definition of $\beta=\left\{\beta_{-1}, \beta_{0}, \ldots, \beta_{L-1}, \beta_{L}\right\}$ in the previous subsection, the problem is to find the optimum $\beta$, such that $p_{\text {succ }}$ is maximized ${ }^{4}$ :

$$
p_{\text {succ }}=\sum_{i=0}^{L-1}\left(\beta_{i}-\beta_{i+1}\right) e^{-\beta_{i}} .
$$

By differentiating with respect to $\beta_{i}$ for $0 \leq i \leq L-1$, we will have:

$$
\beta_{i+1}-\beta_{i}=e^{\beta_{i}-\beta_{i-1}}-1 .
$$

Substituting this in (12), we will have, $p_{\text {succ }}=e^{-\beta_{L-1}}$. Equation (13), can be easily solved and the final solution to the problem can be expressed as follows. Define the sequence $\left\{d_{i}\right\}_{i=0}^{\infty}$, such that $d_{0}=1$ and $d_{k}=1-e^{-d_{k-1}}$ for $k>0$. The optimum values of $\zeta, p_{\text {succ }}$ and $r$ can be expressed as:

$$
\begin{gathered}
\zeta_{i}=\frac{d_{i}}{\sum_{j=0}^{L-1} d_{j}}, \\
p_{s u c c}=e^{-d_{L-1}}, \\
r_{o p t}=\left(1-\frac{p_{\text {succ }}}{\sum_{j=0}^{L-1} d_{j}}\right)^{-1} .
\end{gathered}
$$

It is easy to show that, $\lim _{i \rightarrow \infty} d_{i}=0$. Therefore, $\lim _{L \rightarrow \infty} p_{\text {succ }}=1$.

Fig. 2 shows optimum throughput and backoff factor for different number of power levels. It should be noted that the

\footnotetext{
${ }^{4}$ Note that, $\beta_{-1}$ and $\beta_{L}$ are fixed.
}

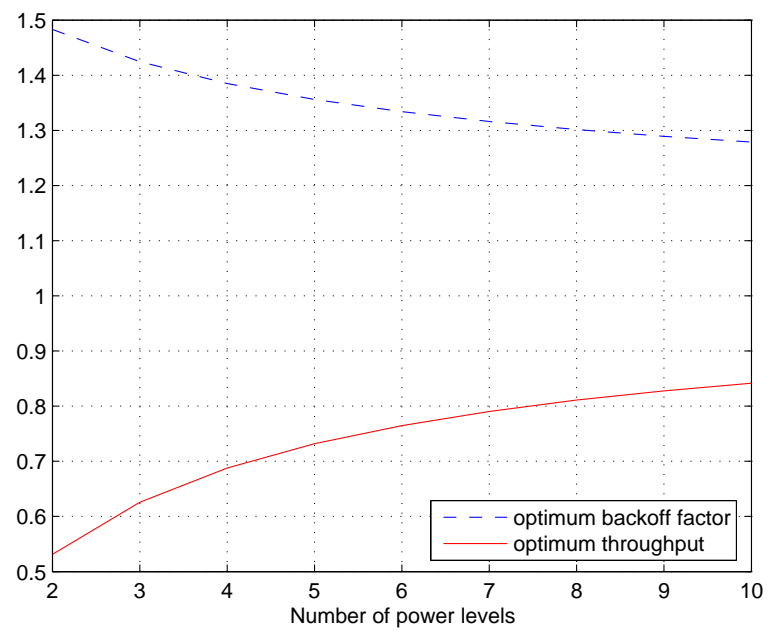

Fig. 2. Optimum throughput and optimum backoff factor for different number of power levels.

optimum values of throughput derived here are exactly the same as the optimum throughput values of [1]. This can be justified as follows. In [1], the author has assumed a Poisson model for network load, which is distributed over $L$ possible transmission powers according to a predetermined scheme. In fact, the traffic of the EB model becomes a Poisson random variable with the average value of $N p_{t}$, when the number of nodes tends to infinity. When we let $r$ to be variable, we are solving the same problem of [1], which is to find the optimum scheme of distributing traffic over available power levels.

\section{ORPSA Dependence on the Number of Nodes}

As mentioned earlier, we have mainly focused on the optimization problem for the case of infinite number of nodes, where we do not have any information of the number of nodes present in the network. In this subsection, we will solve the problem for a given number of nodes $N$, and will compare the throughput values of this locally optimized algorithm with the throughput of the ORPSA derived in the previous subsection. Due to the inefficiency of fixed backoff factor, only variable backoff factors will be considered hereafter. By defining $\beta_{i}^{\prime}=p_{t} \sum_{j=i}^{L-1} \zeta_{j}, \beta_{-1}^{\prime}=1$ and $\beta_{L}^{\prime}=0$, from (2) and (3), $p_{\text {succ }}$ can be expressed as:

$$
p_{\text {succ }}=N \sum_{i=0}^{L-1}\left(\beta_{i}^{\prime}-\beta_{i+1}^{\prime}\right)\left(1-\beta_{i}^{\prime}\right)^{N-1} .
$$

By differentiating with respect to $\beta_{i}^{\prime}$, for $0 \leq i \leq L-1$, we will have:

$$
\beta_{i}^{\prime}-\beta_{i+1}^{\prime}=\frac{1-\beta_{i}^{\prime}}{N-1}\left(1-\left(\frac{1-\beta_{i-1}^{\prime}}{1-\beta_{i}^{\prime}}\right)^{N-1}\right),
$$

for $0 \leq i \leq L-1$. After solving the above equations, the optimum throughput can be calculated from (14) and optimum PMF can be expressed as $\zeta_{i}=\left(\beta_{i}^{\prime}-\beta_{i+1}^{\prime}\right) / \beta_{0}^{\prime}$. Also, noting that $p_{t}=\beta_{0}^{\prime}$, it is obvious from (1) and (3) that:

$$
p_{\text {succ }}=\frac{N \beta_{0}^{\prime}(r-1)}{r-W_{0} \beta_{0}^{\prime} /\left(2-\beta_{0}^{\prime}\right)},
$$




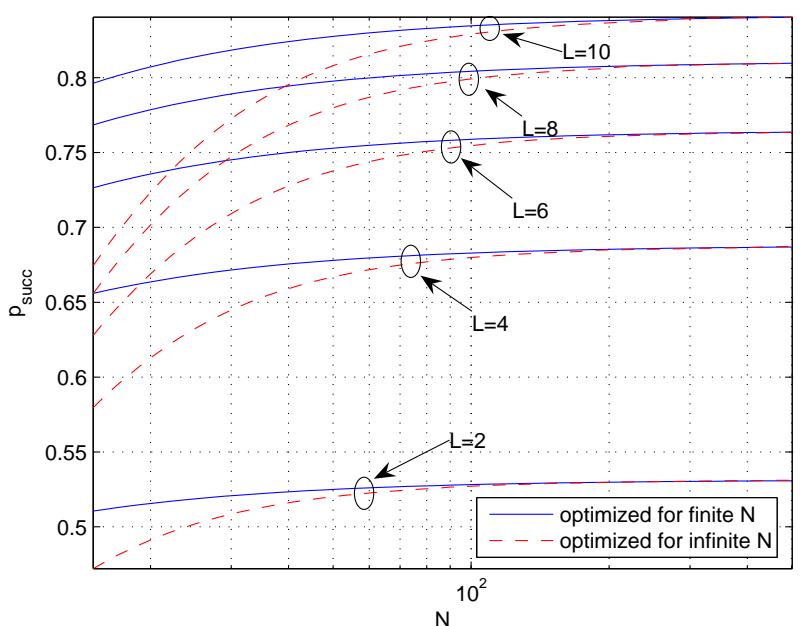

Fig. 3. Comparison of the performance of the optimized algorithm for specific number of nodes with the case where the PMF and backoff factor are optimized for infinite number of nodes.

from which, the amount of optimum backoff factor, $r_{\text {opt }}$ can be calculated. Fig. 3 compares the throughput values, for the case of optimized PMF and backoff factor for a specific value of $N$, with the case where the PMF and backoff factor optimized for infinite $N$ are used. As it is shown in Fig. 3, for large enough number of nodes, the ORPSA optimized for infinite $N$ leads to very similar results obtained by the locally optimized algorithm.

\section{ORPSA with CONSTRAined POWER Budget}

In this section, we will study the problem of ORPSA with limited average power. First, we will derive the optimum power-throughput characteristic based on the assumption of perfect capture model and make a comparison with the powerthroughput characteristic of a random power selection algorithm which uses uniform PMF for power selection. Then, we will introduce an approximate method to conform the perfect model results to SIR-based model.

We will consider a set of logarithmically equi-spaced power levels, $\left\{1, R, R^{2}, \ldots, R^{L-1}\right\}$, where $R$ is the power step. The reasons for choosing such power set are as follows:

- The goal is to study the effect of increasing the number of power levels in the power-throughput characteristic. Therefore, we need the power levels to be clearly discernible from the view of capture capability. This feature does not take place when the power levels are linearly equi-spaced.

- It has been shown that linearly equi-spaced power levels are too far from optimum power levels, while logarithmically equi-spaced power levels can be considered as sub-optimum solutions to the problem of optimum power levels with the model described in [3].

- Logarithmically equi-spaced power levels have been considered for practical system designs. For example, in initialization phase of 802.16 systems [14], the transmission power of RNG-REQ packet is increased in an exponential manner until an ACK is received. 5

\section{A. Optimum Power-Throughput Characteristic}

In this subsection, we will present the power-throughput characteristic of ORPSA with limited average power. For probability of collision, we will use (2) which has formulated the probability of collision with perfect model. By adopting the definition of $\beta$ from III.A and by combining (3), (4) and (7), we will have:

$$
\begin{gathered}
p_{\text {succ }}=\sum_{i=0}^{L-1}\left(\beta_{i}-\beta_{i+1}\right) e^{-\beta_{i}} \\
P_{a v} p_{\text {succ }}=\sum_{i=0}^{L-1}\left(\beta_{i}-\beta_{i+1}\right) R^{i} .
\end{gathered}
$$

Based on these equations, our purpose is maximizing $p_{\text {succ }}$ for a fixed value of $P_{a v}$, which is equivalent to maximizing (16), while (17) holds:

$$
\begin{gathered}
\sum_{i=0}^{L-1}\left(\beta_{i}-\beta_{i+1}\right) R^{i} \\
\sum_{i=0}^{L-1}\left(\beta_{i}-\beta_{i+1}\right) e^{-\beta_{i}}=\sum_{i=0}^{L-1}\left(\beta_{i}-\beta_{i+1}\right) R^{i} / P_{a v} .
\end{gathered}
$$

By adopting Lagrange's method:

$$
\begin{gathered}
\nabla\left\{\sum_{i=0}^{L-1}\left(\beta_{i}-\beta_{i+1}\right) e^{-\beta_{i}}-\sum_{i=0}^{L-1}\left(\beta_{i}-\beta_{i+1}\right) R^{i} / P_{a v}\right\} \\
\propto \nabla\left\{\sum_{i=0}^{L-1}\left(\beta_{i}-\beta_{i+1}\right) R^{i}\right\},
\end{gathered}
$$

and after some manipulations, we will get to the following system of equations:

$$
\begin{gathered}
\beta_{i+1}=e^{\beta_{i}-\beta_{i-1}}+\lambda R^{i} e^{\beta_{i}}+\beta_{i}-1, \quad 0 \leq i \leq L-1 \\
\sum_{i=0}^{L-1}\left(\beta_{i}-\beta_{i+1}\right) R^{i} / P_{a v}=e^{-\beta_{L-1}}-\frac{\lambda\left(R^{L}-1\right)}{R-1} .
\end{gathered}
$$

After solving the above equation for $\lambda$ and $\beta$ (note that $\beta_{-1}=$ $\infty$ and $\beta_{L}=0$ ), throughput can be calculated from (15). Also, the optimum $\zeta$ can be expressed as, $\zeta_{i}=\left(\beta_{i}-\beta_{i+1}\right) / \beta_{0}$, and optimum backoff factor will be, $r_{\text {opt }}=1 /\left(1-p_{\text {succ }} / \beta_{0}\right)$.

Fig. 4 shows the power-throughput characteristic for $2 \leq$ $L \leq 6$, with $R=10$. It should be noted that the powerthroughput characteristic is defined as a function of power budget instead of $P_{a v}$ and the maximum achievable throughput with the average power lower than a determined power budget has been considered as the performance criteria. Also, it should be clear from the optimization process that, the value of power step $(R)$ has a direct effect on the power-throughput characteristic. We will get back to this issue in IV.B.

\footnotetext{
${ }^{5}$ Although the power step has been left as a design parameter in IEEE 802.16 standard, such power increasing capability has mainly been considered to serve as a ranging mechanism rather than throughput improvement algorithm which may exploit the "capture effect".
} 


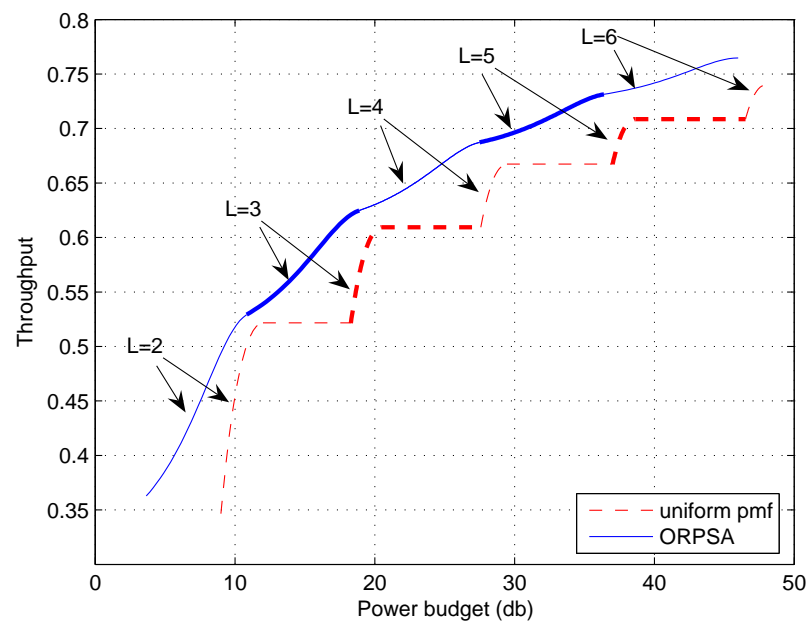

Fig. 4. Optimum power-throughput characteristic and comparison with characteristic of a random algorithm which uses uniform PMF $(R=10)$.

As it is evident from Fig. 4, the main disadvantage of ORPSA is its high power cost for throughput improvement. This cost increases with the number of power levels. For example, for $L=2$ throughput improvement is about 0.023 per $1 d b$ increase in power budget while it is only 0.003 for $L=6$. Therefore, ORPSAs are only appealing with small number of power levels.

In order to show how ORPSA performs in comparison with other random power selection algorithms, we will derive the power-throughput characteristic of a power selection algorithm which uses uniform PMF for power selection. From (2),(3),(4) and (6), and by considering $\zeta_{i}=1 / L$, we will have:

$$
\begin{gathered}
P_{a v} p_{\text {succ }}=\frac{N p_{t}\left(R^{L}-1\right)}{L(R-1)}, \\
P_{a v}=\frac{\left(R^{L}-1\right)\left(e^{N p_{t} / L}-1\right)}{(R-1)\left(1-e^{-N p_{t}}\right)} .
\end{gathered}
$$

For a fixed $P_{a v}, p_{s u c c}$ can be calculated from the above equations. The power-throughput characteristic of this algorithm has also been shown in Fig. 4. As can be verified in this figure, the required power budget can be significantly reduced by use of the optimum algorithm.

\section{B. SIR-based Capture Model}

The optimization process presented in IV.A is based on perfect capture model. The reason is that the formulation of probability of collision with SIR-based model is too complex to be considered in an analytical optimization problem (see [4]). On the other hand, the perfect capture model seems too simplistic to be considered for practical system designs and by considering such capture model, our optimization problem in IV.A has been completely independent of the capture ratio.

In order to highlight the drawbacks of the earlier results, the power-throughput characteristic of ORPSA, introduced in IV.A, has been shown for three distinct power steps in Fig. 5. As can be observed, for a fixed power budget, as the power steps gets smaller, the number of power levels increases and

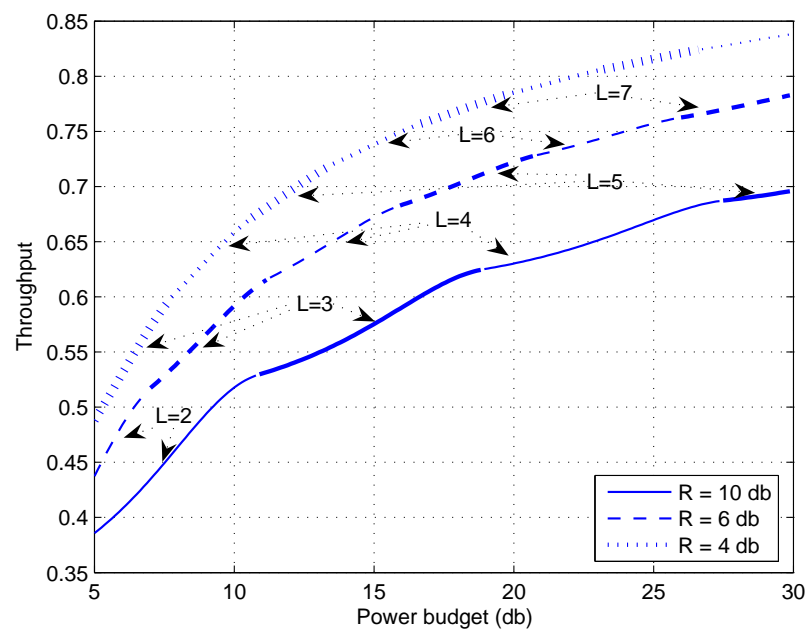

Fig. 5. ORPSA characteristic for different power steps. Very high throughput values can be achieved by using small power steps and considerably low power budget costs.

higher throughput values are achieved. In other words, it is possible to get very high throughput values by almost paying nothing from the average power point of view.

This superficial contradiction stems from the fact that, with small power steps the perfect model capture scenarios rarely comply with the capture condition of SIR-based model. For example, assuming $S I R_{\min }=4, R=3$ and perfect capture model, a packet with power level of $P_{1}=R=3$ will be able to capture the channel against any number of packets with power level of $P_{0}=1$, however, it cannot capture the channel in presence of even one packet from $P_{0}$ level, when SIR-based model is considered. On the other hand, if $R=10$ is considered as the power step, a packet with power $P_{1}=10$ will be able to capture the channel in presence of two or less packets from $P_{0}$ and a packet from $P_{2}=R^{2}=100$ can capture the channel against 25 packets from $P_{0}$ and many other combinations of packets from $P_{0}$ and $P_{1}$. Therefore, noting that in presence of the backoff algorithm the collision size (i.e. the number of simultaneously transmitted packets in one slot) is considerably small, most of the capture scenarios of the perfect model will comply with SIR-based model, if large enough power steps are used. For instance, in the above example, if the backoff algorithm somehow ensures that the collision size in not greater than three, the perfect and SIRbased model will be equivalent for $R=10$.

These observations show that perfect model results are not directly applicable and, therefore, we will introduce an approximate method which will help us to translate the results of perfect model to SIR-based model. Based on this approach, we will be able to present sub-optimum random power selection algorithms for SIR-based capture model. This sub-optimum approach will yield sub-optimum power steps, number of power levels, PMFs and backoff factors for a given power budget constraint. In order to verify the accuracy of analysis, the sub-optimum power-throughput characteristic will be compared with simulation results. Before continuing 
the discussion, two key points should be mentioned.

i) The sub-optimum power-throughput characteristic which will be presented by the end of this subsection, should not be compared with optimum power-throughput characteristics of IV.A for two reasons. First, these characteristics are based on two basically different capture models. A meaningful comparison would be to compare the sub-optimum characteristics with optimum power-throughput characteristics derived based on SIR-based model, which we have been unable to present. Second, the power step has been fixed for perfect model power-throughput characteristics (see Figs. 4 and 5), while in the following method, we will search for the power step which will maximize the throughput. In fact, for the powerthroughput characteristics of IV.A which are based on perfect capture model, an optimum power step does not exist. This is due to the fact that changing power step does not have any effect on the capture condition and as we have discussed earlier, very high throughput values can be achieved by using power steps slightly greater than one.

ii) In the following method, we will only consider power steps which are greater than the capture ratio. Although a case study of smaller power steps have been done for a specific power selection algorithm in [15], these power steps will not be considered here. As discussed earlier, with small power steps the perfect and SIR-based models demonstrate considerably different behaviors and our approximation method will not be applicable anymore.

We will start by estimating the SIR-based throughput based on perfect model throughput. Assume that a packet with power $P_{k}=R^{k}, 0 \leq k \leq L-1$, has captured the channel based on the perfect model. Therefore, no node has made transmission with power equal to or greater than $P_{k}$ and the conditional probability of choosing $P_{i}$ as transmission power becomes $\zeta_{i}^{\prime}=\zeta_{i} / \sum_{j=0}^{k-1} \zeta_{j}$, for $0 \leq i \leq k-1$. Consequently, the probability of $m_{i}$ nodes transmitting with $P_{i}$, for $0 \leq i \leq k-1$, will be:

$$
\begin{gathered}
\operatorname{pr}\left\{\underline{m} \mid \text { perfect }_{k}\right\} \\
=\frac{N !}{\left(N-\sigma_{\underline{m}}\right) ! \prod_{i=0}^{k-1} m_{i} !}\left(1-p_{t}\right)^{N-\sigma_{\underline{m}}} \prod_{i=0}^{k-1}\left(p_{t} \zeta_{i}^{\prime}\right)^{m_{i}},
\end{gathered}
$$

where $\sigma_{\underline{m}}=\sum_{i=0}^{k-1} m_{i}$. For the case of infinite number of nodes, by using (5), we will get:

$$
\operatorname{pr}\left\{\underline{m} \mid \operatorname{perfect} f_{k}\right\}=\prod_{i=0}^{k-1} \frac{\lambda_{i}^{m_{i}}}{m_{i} !} e^{-\lambda_{i}},
$$

where $\lambda_{i}=N p_{t} \zeta_{i}^{\prime}$.

By defining $\rho_{k}$, for $0 \leq k \leq L-1$, as the conditional probability of capture with SIR-based model, given that a perfect model capture has been made by a packet with power $P_{k}=R^{k}$, we will have:

$$
\rho_{k}=\operatorname{pr}\left\{S I R_{k} \mid \operatorname{perfect}_{k}\right\}=\sum_{\underline{m} \in M_{k-1}} \prod_{i=0}^{k-1} \frac{\lambda_{i}^{m_{i}}}{m_{i} !} e^{-\lambda_{i}},
$$

for $1 \leq k \leq L-1$, where,

$$
M_{k-1}=\left\{\left(m_{0}, m_{1}, \ldots, m_{k-1}\right) \mid \sum_{i=0}^{k-1} m_{i} R^{i}<\frac{R^{k}}{S I R_{\min }}\right\} .
$$

Note that $\rho_{0}=1$. The equation (18) does not seem to reduce to a simple analytical expression since all the vectors in $M_{k-1}$ are required to be known. Define $M_{k-1}^{\prime}$ for $1 \leq k \leq L-1$, as follows:

$$
\begin{aligned}
& \underline{m} \in M_{k-1}^{\prime} \Leftrightarrow \\
& \left(m_{k-1}=\lfloor\Delta\rfloor \wedge m_{i}=0, \text { for } 0 \leq i<k-1\right) \quad \vee \\
& \left(0 \leq m_{k-1} \leq\lfloor\Delta\rfloor-1 \wedge 0 \leq m_{i} \leq\lfloor R\rfloor-1 \text {, for } 0 \leq i<k-1\right) \text {, }
\end{aligned}
$$

where $R$ is the power step and $\Delta=R / S I R_{\min }$. It is easy to show that, $M_{k-1}^{\prime} \subseteq M_{k-1}$. Therefore,

$$
\begin{gathered}
\rho_{k} \geq 1-\epsilon_{k}=\sum_{\underline{m} \in M_{k-1}^{\prime}} \prod_{i=0}^{k-1} \frac{\lambda_{i}^{m_{i}}}{m_{i} !} e^{-\lambda_{i}}=\frac{\lambda_{k-1}^{\lfloor\Delta\rfloor}}{\lfloor\Delta\rfloor !} e^{-N p_{t}} \\
+\left(\prod_{i=0}^{k-2} \sum_{m_{i}=0}^{\left\lfloor R^{-1}\right.} \frac{\lambda_{i}^{m_{i}}}{m_{i} !} e^{-\lambda_{i}}\right) \sum_{m_{k-1}=0}^{\lfloor\Delta\rfloor-1} \frac{\lambda_{k-1}^{m_{k-1}}}{m_{k-1} !} e^{-\lambda_{k-1}} .
\end{gathered}
$$

It should be noted that $\epsilon_{0}=0$. For the average conditional probability of SIR-based capture, conditioned on perfect capture, we should calculate the average of $\rho_{k}$ over $k$ :

$$
\begin{gathered}
\rho=\sum_{k=0}^{L-1} \frac{\rho_{k} \cdot p r\left\{\text { power }=R^{k}\right\} \cdot p r\left\{\text { success with } R^{k}\right\}}{p r\{\text { success }\}} \\
=\frac{\sum_{k=0}^{L-1} \rho_{k} \zeta_{k} e^{-N p_{t} \sum_{j=k}^{L-1} \zeta_{j}}}{\sum_{k=0}^{L-1} \zeta_{k} e^{-N p_{t} \sum_{j=k}^{L-1} \zeta_{j}}} .
\end{gathered}
$$

Therefore,

$$
1-\rho \leq \frac{\sum_{k=0}^{L-1} \epsilon_{k} \zeta_{k} e^{-N p_{t} \sum_{j=k}^{L-1} \zeta_{j}}}{\sum_{k=0}^{L-1} \zeta_{k} e^{-N p_{t} \sum_{j=k}^{L-1} \zeta_{j}}} .
$$

The value of $1-\rho$ represents the difference between two capture models. For example, $\rho=0.9$ means that 90 percent of the cases which have resulted in successful transmissions with perfect model would have been successful if SIR-based model was considered. Fig. 6 shows the maximum upper bound value of (20) within the power budget ranges considered for each number of power levels for $R=10$ (see Fig. 4). As $\Delta$ gets larger, the space between power step $(R)$ and capture ratio $\left(S I R_{\min }\right)$ increases and the two models will perform more similarly.

Considering $\rho$ as a measure of closeness of perfect and SIRbased capture models, we will use the following approximation for SIR-based throughput,

$$
\text { Throughput } \approx \rho \times p_{\text {succ }},
$$

where by $p_{\text {succ }}$ we mean the perfect model throughput. We have considered the search range of $\left[S I R_{\min } S I R_{\min }+10\right]_{d b}$ for the power step. For a given value of $P_{a v}$ and for any $R$ in this search range, the value of $L$ is extracted from the corresponding optimum power-throughput characteristics of IV.A. For example, for $P_{a v}=25 d b$ and $R=10, L$ will be 4 . For given $R$ and $L$ values, the corresponding values of $\underline{\zeta}$ and $\beta_{0}=N p_{t}$ are determined from the optimization process presented in IV.A. Incorporating these with (19) and (20) and considering the inequalities as approximate values, $\rho$ will be determined and the throughput is calculated from (21). 


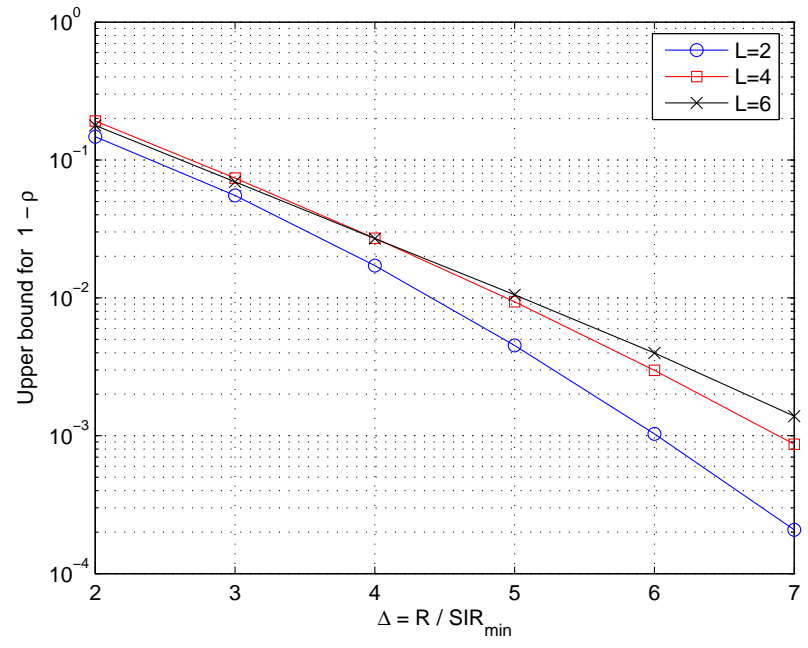

Fig. 6. Upper bound for maximum difference between the power-throughput characteristic of ORPSA based on perfect and SIR-based capture model.

TABLE I

Sub-optimum Power Steps, Number of Power Levels, PMF AND Backoff Factor For Different Power Budget Values; $S I R_{\text {min }}=6.0 \mathrm{db}$

\begin{tabular}{|c|c|c|c|c|c|}
\hline Power budget $(\mathrm{db})$ & 5 & 15 & 25 & 35 & 45 \\
\hline \hline Power step $(\mathrm{db})$ & 6.000 & 12.026 & 12.997 & 12.997 & 12.026 \\
\hline No. of levels & 2 & 3 & 4 & 4 & 6 \\
\hline PMF & 0.8389 & 0.6194 & 0.4830 & 0.4279 & 0.3617 \\
& 0.1611 & 0.3637 & 0.3041 & 0.2701 & 0.2286 \\
& & 0.0169 & 0.2123 & 0.1962 & 0.1694 \\
& & & 0.0006 & 0.1058 & 0.1346 \\
& & & & & 0.1048 \\
& & & & 0.0010 \\
\hline Backoff factor & 1.8801 & 1.5128 & 1.4337 & 1.4120 & 1.3604 \\
\hline Throughput & 0.4063 & 0.5354 & 0.6217 & 0.6761 & 0.7127 \\
\hline
\end{tabular}

Finally, the power step which results in highest throughput is considered as the sub-optimum power step for the given power budget and the corresponding values of $L, \underline{\zeta}$ and $r$ are considered as the sub-optimum values of number of power levels, PMF and backoff factor. Table I summarizes these sub-optimum values and the corresponding sub-optimum throughput values for different values of power budget.

In order to verify the presented sub-optimum powerthroughput characteristic for SIR-based model, we have simulated networks of 100 and 1000 nodes, which use the presented sub-optimum values of $R, L, \zeta$ and $r$ for power selection and contention resolution. The initial contention window size $\left(W_{0}\right)$ and capture ratio $\left(S I R_{\min }\right)$ have been 16 and $6.0 \mathrm{db}$, respectively. Each power budget and throughput pair has been recorded after 100,000, 000 time slots, based on SIR-based capture model. Once again, it should be noted that the powerthroughput characteristic is defined as a function of power budget instead of $P_{a v}$ and the maximum achievable throughput with the average power lower than a determined power budget has been considered as the output of our simulation. Fig. 7 compares the sub-optimum power-throughput characteristic with the simulation results. We observe that the simulation

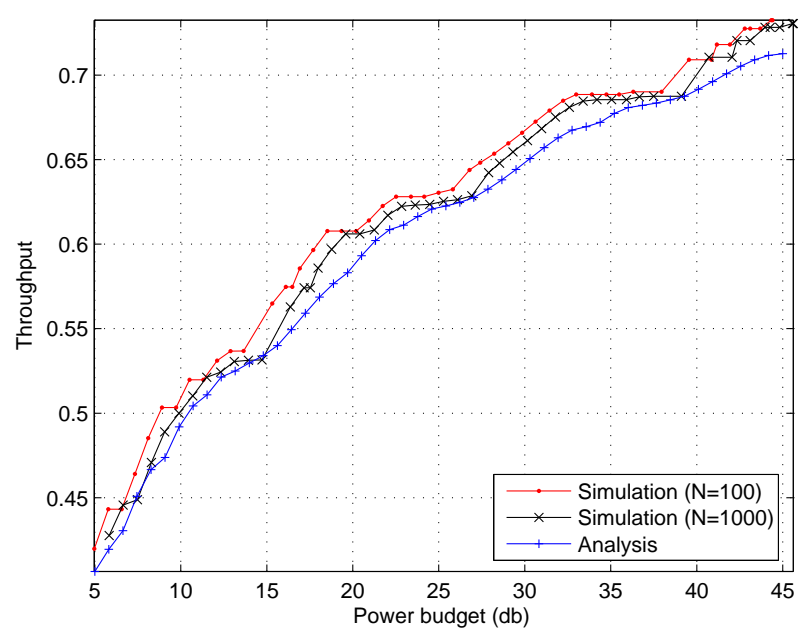

Fig. 7. Comparison of sub-optimum power-throughput characteristic for random power section algorithms based on SIR-based capture model with the simulation results for $N=100$ and $1000\left(W_{0}=16, S I R_{\min }=6.0 \mathrm{db}\right)$.

results lie above the sub-optimum characteristic, which verifies our approach in introducing $\rho$ and presentation of lower bound approximation for the behavior of SIR-based model. Moreover, as the number of nodes increases, the simulated characteristic gets closer to the sub-optimum characteristic derived assuming the infinite nodes case.

At this point, we will finish discussion of the random power selection algorithms and in the remainder of this paper, we will introduce the possibility of using deterministic power selection algorithms instead of random techniques. The main advantage of these algorithms is that they do not require any random power generator blocks. In contrast to random algorithms where the selected power is independent of the transmission history of packets, with deterministic approach the transmission power will be a function of the number of collisions encountered. In section VI the system model will be presented. The unconstrained and constrained power scenarios will be discussed in sections VII and VIII, based on which sub-optimum deterministic power selection and suboptimum power-throughput characteristics will be presented. It is important to note that these results will be based on perfect capture model and from this point of view are comparable with the results of sections III and IV.A. An approach similar to IV.B may be used to adapt the results for SIR-based capture model.

\section{Modeling of Exponential Backoff With \\ Deterministic Power Selection Algorithms}

Assume a set of available power levels $\mathbf{P}=$ $\left\{P_{0}, P_{1}, \ldots, P_{L-1}\right\}$, where $L$ is the number of power levels. By a deterministic power selection algorithm, we mean a function from the set of all nonnegative integers to $\mathbf{P}$. This function, represented by $P^{(n)}$, determines the transmission power at backoff state $n$, i.e. the (re)transmission power of a packet which has had $n$ successive collisions.

The EB model of [13] has been modified to describe the deterministic power selection scenario (Fig. 8). A node in state 
$i$, will eventually transmit with power level equal to $P^{(i)}$ and will experience collision with the probability $\alpha\left(P^{(i)}\right)$, leading to state $i+1$, otherwise the node will get back to state 0 . The definitions of $W_{n}$ and $r$ are the same as in section II.

The following theorem presents the basic relations which we will use in analysis of deterministic algorithms.

Theorem 2: Consider a network of $N$ nodes which are using the algorithm depicted in Fig. 8 for contention resolution and power selection. Define $p_{i}, p_{t}, p_{\text {succ }}$ and $P_{a v}$ as the steady state probability of state $i, i \geq 0$, the average probability of transmission, probability of successful transmission (throughput) and average required power for a successful transmission, respectively. Also, define $\alpha_{j}=\alpha\left(P_{j}\right)$, for $0 \leq j \leq L-1$. We will have:

$$
\begin{gathered}
p_{i}=p_{0} \prod_{j=0}^{i-1} \alpha\left(P^{(j)}\right), \quad i \geq 06 \\
p_{t}=\frac{2 / W_{0}}{\sum_{i=0}^{\infty} p_{i} r^{i}+1 / W_{0}} \\
p_{\text {succ }}=N p_{t} p_{0} \\
P_{a v}=\sum_{i=0}^{\infty} P^{(i)} p_{i} / p_{0} \\
\alpha_{j}=1-\left(1-\sum_{P^{(k)} \geq P_{j}} p_{t} p_{k}\right)^{N-1}, \quad 0 \leq j \leq L-1 \\
\text { Proof: } \quad
\end{gathered}
$$

(22) is obvious from the presented model.

(23) can be proven following exactly the same approach presented in [13].

The probability of successful transmission is the probability that a node which may transmit in any state with probability $p_{t} p_{i}, i \geq 0$, makes transmission and does not face collision. Therefore:

$$
p_{\text {succ }}=N \sum_{i=0}^{\infty} p_{t} p_{i}\left(1-\alpha\left(P^{(i)}\right)\right)=N p_{t} \sum_{i=0}^{\infty}\left(p_{i}-p_{i+1}\right) .
$$

This will prove (24).

A packet which has been finally transmitted successfully at state $i$ with probability of $\left(1-\alpha\left(P^{(i)}\right)\right) \prod_{j=0}^{i-1} \alpha\left(P^{(j)}\right)$, has experienced a transmission history with $P^{(0)}, P^{(1)}, \ldots$ and $P^{(i)}$. Therefore, noting (22), the average required power for successful transmission will be:

$$
\begin{gathered}
P_{a v}=\sum_{i=0}^{\infty}\left(\left(1-\alpha\left(P^{(i)}\right)\right) \prod_{j=0}^{i-1} \alpha\left(P^{(j)}\right)\right)\left(\sum_{k=0}^{i} P^{(k)}\right) \\
=1 / p_{0} \sum_{i=0}^{\infty}\left(p_{i}-p_{i+1}\right)\left(\sum_{k=0}^{i} P^{(k)}\right)=\sum_{i=0}^{\infty} P^{(i)} p_{i} / p_{0} .
\end{gathered}
$$

This proves (25).

(26) has simply formulated the probability of collision based on perfect capture model. The probability of success with transmission power of $P_{j}$ is the probability that no simultaneous packet has been transmitted with power equal to or greater than $P_{j}$.

${ }^{6}$ Define, $\prod_{0}^{-1}$ to be 1 .

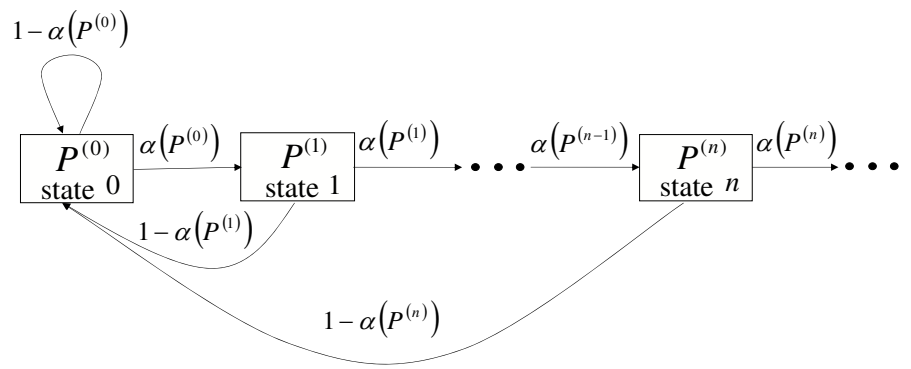

Fig. 8. EB model with deterministic power selection algorithm. A node in state $i$, transmits with $P^{(i)}$ and faces collision with probability $\alpha P^{(i)}$.

For the case of infinite number of nodes, we will have:

$$
\lim _{N \rightarrow \infty} p_{t}=0 .
$$

Also, assuming that $\lim _{i \rightarrow \infty} P^{(i)}$ exists and by defining $K$ as the least nonnegative integer such that $P^{(j)}=P^{(K)}$, for all $j>K$, we will have:

$$
\lim _{N \rightarrow \infty} \alpha\left(P^{(K)}\right)=\frac{1}{r} .
$$

For proof of (27), assume that $\lim _{N \rightarrow \infty} p_{t}>0$, then from (26), $\alpha_{j}=1,0 \leq j \leq L-1$, which is a contradiction.

In order to prove (28), we should note that the denominator of (23) contains $\sum_{i=K}^{\infty} p_{i} r^{i}=p_{K} r^{K} \sum_{0}^{\infty}\left(r \alpha\left(P^{(K)}\right)\right)^{i}$, and other finite terms. It should be obvious that for a finite $N, \alpha\left(P^{(K)}\right)<1 / r$, otherwise $p_{t}$ will be 0 , and from (26), $\alpha_{j}=0,0 \leq j \leq L-1$, which is a contradiction. Therefore, $\lim _{N \rightarrow \infty} \alpha\left(P^{(K)}\right) \leq 1 / r$. Also, from (27) and the extracted term from the denominator of (23), it is obvious that $\lim _{N \rightarrow \infty} \alpha\left(P^{(K)}\right) \geq 1 / r$. Combining these, the proof of (28) will be complete.

By combining (26) and (27) and by defining $\gamma_{i}=N p_{t} p_{i}$, the expression of the probabilities of collision can be simplified to:

$$
\alpha_{j}=1-\exp \left(-\sum_{P^{(k)} \geq P_{j}} \gamma_{k}\right), \quad 0 \leq j \leq L-1,
$$

where, $\gamma_{k}=\gamma_{0} \prod_{i=0}^{k-1} \alpha\left(P^{(i)}\right)$.

By deriving the above basic relations, we will go through the problem of Optimum Deterministic Power Selection Algorithm (ODPSA) in sections VI and VII.

\section{ODPSA With UnConstrained Power Budget}

In this section, we will discuss the optimum deterministic algorithm without considering any limit on the average power. Our goal is to find the power sequence $\left\{P^{(n)}\right\}_{n=0}^{\infty}$ which satisfies (29) and yields optimum throughput. However, we were unable to derive even approximate pattern of optimum power sequence based on (29). Our further investigations showed that there are many power sequences that yield approximately same throughput values, but do not have considerably similar patterns. In other words, the throughput of the deterministic algorithms is not a well-behaved function of the pattern of the power sequences. Therefore, we have taken an indirect 
approach to derive optimum power deterministic power selection algorithms. First, we show that the throughput of ORPSA is an upper bound for the throughput of ODPSA. Then, we will present our search method for ODPSA based on which sub-optimum algorithms (sub-ODPSAs) will be derived.

Defining, $\beta_{i}=\sum_{P^{(j)} \geq P_{i}} \gamma_{j}$ and noting (29), we will have:

$$
\begin{gathered}
\sum_{i=0}^{L-1}\left(\beta_{i}-\beta_{i+1}\right) e^{-\beta_{i}}=\sum_{i=0}^{L-1}\left(\sum_{P^{(j)}=P_{i}} \gamma_{j}\right) \exp \left(-\sum_{P^{(k)} \geq P_{i}} \gamma_{k}\right) \\
=\sum_{i=0}^{L-1} \sum_{P^{(j)}=P_{i}} \gamma_{j}\left(1-\alpha_{i}\right)=\sum_{i=0}^{L-1} \sum_{P^{(j)}=P_{i}}\left(\gamma_{j}-\gamma_{j} \alpha\left(P^{(j)}\right)\right) \\
=\sum_{i=0}^{L-1} \sum_{P^{(j)}=P_{i}}\left(\gamma_{j}-\gamma_{j+1}\right)=\sum_{j=0}^{\infty}\left(\gamma_{j}-\gamma_{j+1}\right)=\gamma_{0} .
\end{gathered}
$$

Therefore, for the case of unlimited power budget, we have the same relation for throughput as (12) in III.B, which has formulated the throughput for random power selection scenario:

$$
\gamma_{0}=\sum_{i=0}^{L-1}\left(\beta_{i}-\beta_{i+1}\right) e^{-\beta_{i}} .
$$

Hence, with unlimited power budget, the throughput of OPRSA will be an upper bound for the throughput of ODPSA. By adopting the definition of $\left\{d_{i}\right\}_{i=0}^{\infty}$ from section III.B, the upper-bound-condition values of $\alpha_{i}$ and $\gamma_{i}$, can be expressed as follows:

$$
\begin{gathered}
\gamma_{0}=e^{-d_{L-1}}, \\
\alpha_{i}=1-e^{-\sum_{j=i}^{L-1} d_{j}}, \\
\sum_{P^{(j)}=P_{i}} \gamma_{j}=d_{i},
\end{gathered}
$$

for $0 \leq i \leq L-1$. The next step toward ODPSA is to find the power sequence $\left\{P^{(n)}\right\}_{n=0}^{\infty}$, which satisfies (31), (32) and (33). Instead of searching for such a power sequence, which does not necessarily exist, we can consider the upper-boundcondition collision probabilities $\alpha_{i}$ of (32), and throughput $\gamma_{0}$ of (31), and search for a power sequence $\left\{P^{\prime(n)}\right\}_{n=0}^{\infty}$, for which the values of $\sum_{P^{\prime(j)}=P_{i}} \gamma_{j}^{\prime}$ for $0 \leq i \leq L-1$, are closest to the upper-bound-condition values of (33). If we define $D$ as the depth of the search ${ }^{7}$, the problem will be to search for $\left\{P^{\prime(n)}\right\}_{n=0}^{D-1}$, such that:

$$
e_{M S E}=\frac{1}{L} \sum_{i=0}^{L-1}\left(d_{i}-\sum_{P^{\prime(j)}=P_{i}}^{j<D} \gamma_{j}^{\prime}\right)^{2}
$$

is minimized, where $\gamma_{j}^{\prime}=\gamma_{0}^{\prime} \prod_{k=0}^{j-1} \alpha\left(P^{\prime(k)}\right)$. Note that $\gamma_{0}^{\prime}=$ $\gamma_{0}$.

Fig. 9 shows an efficient and quick search method, which we have used to achieve results close to ODPSA. In this method, we construct a set of Acceptable Power Sequences (APS), which is initially empty. This set is expanded at each level of the search, by checking the possibility of selecting any of the power levels for the next backoff state (search level). The

\footnotetext{
${ }^{7} \mathrm{By}$ depth of search, we mean the maximum number of backoff states which we will consider in our search.
}

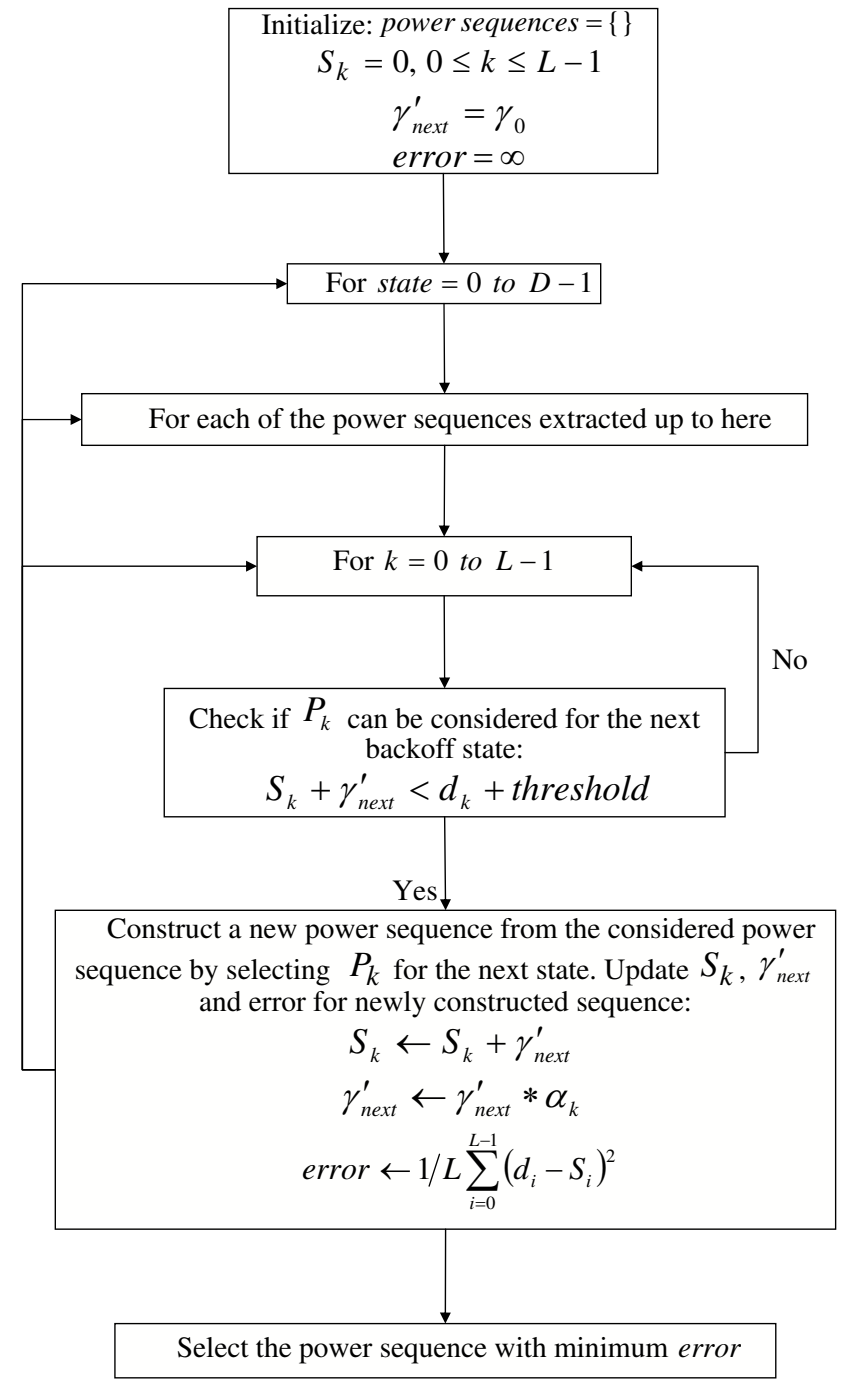

Fig. 9. The proposed search method for sub-ODPSA.

following variables are associated to each of the elements of APS:

- For $k=1,2, \ldots, L-1, S_{k}$ will hold the summation of all $\gamma_{j}^{\prime}$ for all indices $j$ of the power sequence, for which $P^{\prime(j)}=P_{k} . S_{k}$ has been initially set to zero.

- $\gamma_{\text {next }}^{\prime}$ will hold the value of $\gamma^{\prime}$ for the next backoff state or next level of search. In other words, if we are at the level $l$ of search $(0 \leq l \leq D-1), \gamma_{\text {next }}^{\prime}$ will be $\gamma_{0}^{\prime} \prod_{j=0}^{l} \alpha\left(P^{\prime(j)}\right)$. Each time in the search process that the power level of $P_{k}$ is considered for the next backoff state, $\gamma_{n e x t}^{\prime}$ will be added to $S_{k}$ and updated to $\gamma_{n e x t}^{\prime} \alpha_{k}$. The initial value of $\gamma_{\text {next }}^{\prime}$ is set to $\gamma_{0}^{\prime}$ which is known from (31).

- error which is used for choosing the power sequence with minimum deviation from upper-bound-condition condition of (33), at the end of the search:

$$
\text { error }=\frac{1}{L} \sum_{i=0}^{L-1}\left(d_{i}-S_{i}\right)^{2} .
$$

At each step of the search process, the elements of the APS 
TABLE II

CONSTRUCTION PROCESS OF APS FOR $L=2$ AND $D=4$; threshold $=0$

\begin{tabular}{|c|c|c|c|}
\hline Level 0 & Level 1 & Level 2 & Level 3 \\
\hline \multirow{18}{*}{$\begin{array}{l}\text { Seq. }=\{0\} \\
S_{0}=0.5315 \\
S_{1}=0 \\
\gamma_{\text {next }}^{\prime}=0.4276 \\
\text { error }=0.3096\end{array}$} & $\{0,0\}$ & $\{0,0,1\}$ & $\{0,0,1,1\}$ \\
\hline & 0.9590 & 0.9590 & $\underline{0.9590}$ \\
\hline & 0 & 0.3440 & $\underline{0.5051}$ \\
\hline & 0.3440 & 0.1612 & $\underline{0.0755}$ \\
\hline & 0.2006 & 0.0424 & $\overline{0.0089}$ \\
\hline & \multirow{13}{*}{$\begin{array}{l}\{0,1\} \\
0.5315 \\
0.4276 \\
0.2003 \\
0.1307\end{array}$} & \multirow{8}{*}{$\begin{array}{l}\{0,1,0\} \\
0.7318 \\
0.4276 \\
0.1612 \\
0.0569\end{array}$} & $\begin{array}{l}\{0,1,0,0\} \\
0.8929\end{array}$ \\
\hline & & & 0.4276 \\
\hline & & & 0.1296 \\
\hline & & & 0.0267 \\
\hline & & & $\{0,1,0,1\}$ \\
\hline & & & 0.7318 \\
\hline & & & 0.5887 \\
\hline & & & 0.0755 \\
\hline & & $\{0,1,1\}$ & $\{0,1,1,0\}$ \\
\hline & & 0.5315 & 0.6253 \\
\hline & & 0.6279 & 0.6279 \\
\hline & & 0.0939 & 0.0755 \\
\hline & & 0.1098 & 0.0702 \\
\hline Seq. $=\{1\}$ & $\{1,0\}$ & $\{1,0,0\}$ & $\{1,0,0,0\}$ \\
\hline$S_{0}=0$ & 0.2490 & 0.4493 & 0.6105 \\
\hline$S_{1}=0.5315$ & 0.5315 & 0.5315 & 0.5315 \\
\hline$\gamma_{\text {next }}^{\prime}=0.2490$ & 0.2003 & 0.1612 & 0.1296 \\
\hline error $=0.5051$ & 0.2871 & 0.1567 & 0.0809 \\
\hline
\end{tabular}

are checked to see if any of the $L$ power levels can be considered for their next backoff state. We have used the following condition for checking this possibility:

$$
S_{k}+\gamma_{\text {next }}^{\prime}<d_{k}+\text { threshold }
$$

The value of threshold is optional and controls the size of search. If the above condition holds, that power level is added to the power sequence to construct a new member of APS and the corresponding values of $S_{k}$ and $\gamma_{n e x t}^{\prime}$ are calculated accordingly. If the condition cannot be satisfied by any of the power levels, the selected power sequence will be deleted from APS. Table II shows the construction process of APS for $L=2$ and $D=4$. The Seq. symbol refers to a power sequence in APS. In order to save the space, the left side symbols of column one have been omitted in other columns of the table. The power sequence with the minimum error after a four level search is underlined in the table.

It should be noted that, this search method does not necessarily give the minimum $e_{M S E}$, since we ignore all power sequences with $S_{k}+\gamma_{\text {next }}^{\prime}>d_{k}+$ thresold, and the algorithm with minimum $e_{M S E}$ may be among these. However, without considering such constraint, the search will be extremely timeconsuming. Moreover, the resulting power sequences will perform very close to the upper bound of optimum throughput as we will see in the remainder of this section. Fig. 10 shows our search results and corresponding error values as defined in (34).

In the next step, we need to extend these power sequences to construct sub-ODPSAs by defining $P^{\prime(n)}=P_{L-1}$ for $n \geq D$,

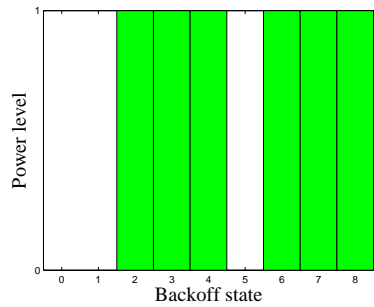

(a)

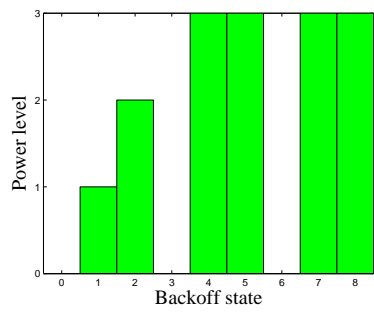

(c)

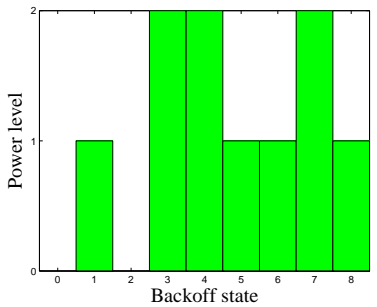

(b)

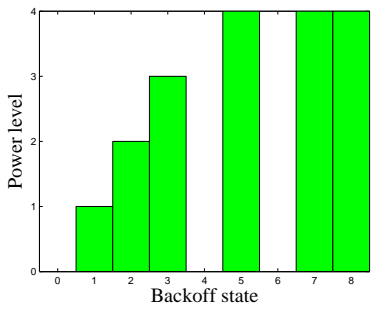

(d)
Fig. 10. Extracted power sequences for $D=9$ : (a) $L=2, e_{M S E}=2.16 \times$ $10^{-5}$, threshold $=0$; (b) $L=3, e_{M S E}=3.24 \times 10^{-5}$, threshold $=0$; (c) $L=4, e_{M S E}=1.41 \times 10^{-4}$, threshold $=0.026 \gamma_{0}$; (d) $L=5$, $e_{M S E}=3.90 \times 10^{-3}$, threshold $=0.142 \gamma_{0}$.

and make exact analysis of these power sequences ${ }^{8}$. We will use the notations $\tilde{\alpha}_{i}$ and $\tilde{\gamma}_{i}$ for sub-ODPSAs, so that these quantities can be distinguished from those of upper bound condition. Noting (29) and defining $\tilde{\alpha}_{L}=0$, the following system of equations should be satisfied:

$$
\frac{1-\tilde{\alpha}_{i}}{1-\tilde{\alpha}_{i+1}}=\exp \left(-\sum_{P^{\prime(j)}=P_{i}} \tilde{\gamma}_{j}\right), \quad 0 \leq i \leq L-1,
$$

which will have the following form after considering $P^{\prime(n)}=$ $P_{L-1}$ for $n \geq D$ :

$$
\begin{gathered}
\ln \left(\frac{1-\tilde{\alpha}_{i}}{1-\tilde{\alpha}_{i+1}}\right)+\sum_{P^{\prime(j)}=P_{i}}^{j<D} \tilde{\gamma}_{j}=0, \quad 0 \leq i \leq L-2 \\
\ln \left(1-\tilde{\alpha}_{L-1}\right)+\sum_{P^{\prime(j)}=P_{L-1}}^{j<D} \tilde{\gamma}_{j}+\frac{\tilde{\gamma}_{D-1} \tilde{\alpha}_{L-1}}{1-\tilde{\alpha}_{L-1}}=0,
\end{gathered}
$$

where $\tilde{\gamma}_{j}=\tilde{\gamma}_{0} \prod_{k=0}^{j-1} \tilde{\alpha}\left(P^{\prime(k)}\right)$. This will lead to $L+1$ unknown variables, $\tilde{\gamma}_{0}, \tilde{\alpha}_{0}, \tilde{\alpha}_{1}, \ldots, \tilde{\alpha}_{L-1}$, with $L$ equations. This is due to the fact that the upper-bound-condition equations (31), (32) and (33) do not give the corresponding value of the backoff factor. Therefore, we will leave $\alpha_{L-1}$ as an optimization parameter. Noting (28), this is equivalent to leaving $\tilde{r}$ as a variable to be optimized.

After solving the above equations, the value of throughput, $\tilde{\gamma}_{0}$, can be calculated. In Fig. 11, these sub-optimum throughput values have been compared with the throughput of ORPSA, which has been shown to be an upper bound for the throughput of ODPSA. As it is evident from Fig. 11,

\footnotetext{
${ }^{8}$ Such a choice is obviously optional. Our choice in favor of $P_{L-1}$ results from the frequent appearance of this power level in higher states of the extracted power sequences (see Fig 10).
} 


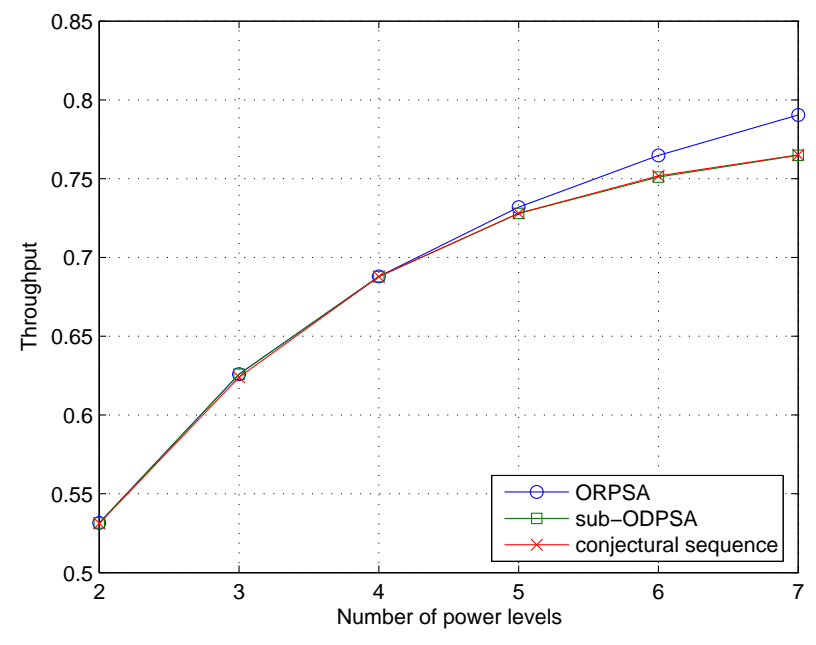

Fig. 11. Comparison of ORPSA throughput, as an upper bound for the throughput of ODPSA, with throughput of sub-ODPSA and conjectural sequence $(D=9)$.

throughput values of sub-ODPSA are very close to throughput of ORPSA, which has been proven to be an upper bound for ODPSA throughput. Therefore, it is obvious that the proposed algorithms will have very similar performance to ODPSA as well.

Although our approach in this section presents a method to drive sub-optimum deterministic power selection algorithms (power sequences), we have not given a general visual pattern of such power sequences. For this purpose, we will present a conjectural sequence for ODPSA. This scheme is depicted in Fig. 12 and has been constructed based on the similarities of the sub-optimum power sequences (see Fig. 10). The throughput of this power selection algorithm can be calculated from similar equations, discussed for sub-optimum algorithms and has been included in Fig. 11.

As mentioned in the beginning of this section, the throughput of deterministic power selection algorithms is not a wellbehaved function of the pattern of the power sequences. However, some general rules can be extracted about the pattern of the optimum power sequences. For example, by considering (33) and noting that $\left\{d_{i}\right\}_{i=0}^{\infty}$ is a decreasing sequence (see III.B), we conclude that $\left\{\sum_{P^{(j)}=P_{i}} \gamma_{j}\right\}_{i=0}^{L-1}$ is decreasing with respect to $i$. Noting that $\left\{\gamma_{j}\right\}_{j=0}^{\infty}$ is a decreasing sequence, one way of satisfying the decreasing trend of $\left\{\sum_{P^{(j)}=P_{i}} \gamma_{j}\right\}$, with respect to $i$, is to use smaller power levels in lower backoff states and larger power levels in higher backoff states. This conclusion justifies the pattern of conjectural sequence in Fig. 12.

\section{ODPSA with Constrained Power Budget}

In this section, we will discuss the power-throughput characteristic of ODPSA. Our purpose is to find a power sequence which has maximum value of throughput for a predetermined value of average power. We will first prove that the characteristic of ORPSA will be an upper bound for that of ODPSA. Next, we will use a search method similar to that

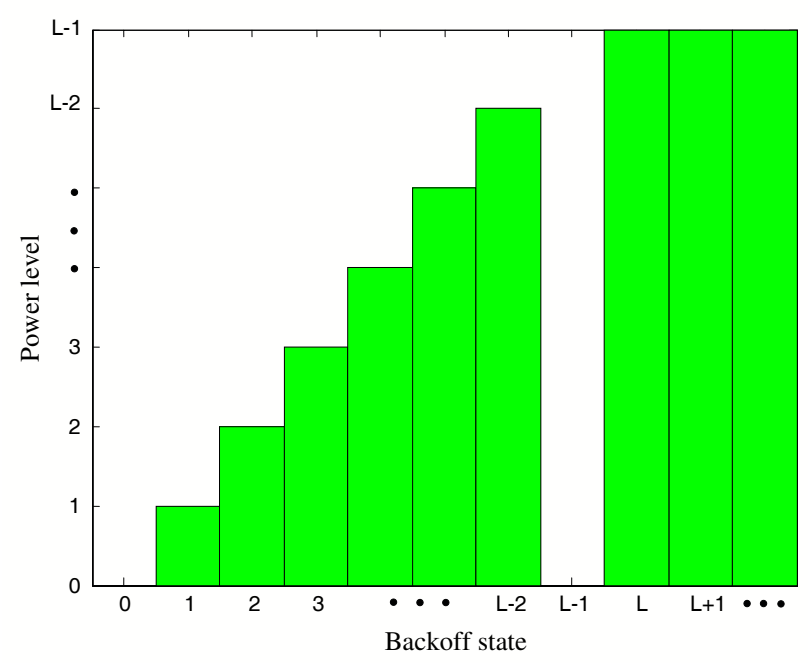

Fig. 12. Conjectural power sequence. The throughput of this algorithm has been compared with the throughput of sub-ODPSA and ORPSA in Fig. 11.

discussed in the previous section which will give the suboptimum characteristic. We will show that this sub-optimum characteristic will perform very close to the upper bound.

From (25) and definition of $\gamma_{i}$ and $\beta_{i}, i \geq 0$, the average power can be expressed as:

$$
\begin{gathered}
P_{a v}=\sum_{i=0}^{\infty} P^{(i)} \gamma_{i} / \gamma_{0}=\sum_{i=0}^{L-1} P_{i}\left(\sum_{P^{(j)}=P_{i}} \gamma_{j} / \gamma_{0}\right) \\
=1 / \gamma_{0} \sum_{i=0}^{L-1}\left(\beta_{i}-\beta_{i+1}\right) P_{i} .
\end{gathered}
$$

As discussed in section IV, we will consider logarithmically equi-spaced power levels, $\mathbf{P}=\left\{1, R, R^{2}, \ldots, R^{L-1}\right\}$, where $R$ is the power step. Therefore, we will have:

$$
\begin{gathered}
\gamma_{0}=\sum_{i=0}^{L-1}\left(\beta_{i}-\beta_{i+1}\right) e^{-\beta_{i}} \\
P_{a v}=1 / \gamma_{0} \sum_{i=0}^{L-1}\left(\beta_{i}-\beta_{i+1}\right) R^{i} .
\end{gathered}
$$

It is evident that the above equations constitute exactly the same optimization problem discussed in IV.A, for limited power budget scenario. Consequently, the power-throughput characteristic of ORPSA can be considered as an upper bound for that of ODPSA. After calculating the upper-boundcondition values of $\gamma_{0}, \alpha_{i}$ and $\sum_{P^{(j)}=P_{i}} \gamma_{j}$ for $0 \leq i \leq L-1$, a search algorithm similar to one shown in Fig. 9 can be used to find power sequences $\left\{P^{\prime(n)}\right\}_{n=0}^{D-1}$, which will be close enough to the upper bound conditions. Then, by repeating the last power level, $P^{\prime(n)}=P^{\prime(D-1)}$ for $n \geq D$, sub-ODPSAs will be constructed. These sub-optimum power sequences should be analyzed in order to reveal exact values of throughput, $\tilde{\gamma}_{0}$, and probabilities of collision, $\tilde{\alpha}_{i}$ for $0 \leq i \leq L-1$.

Considering (35) and (36), the following system of equa- 


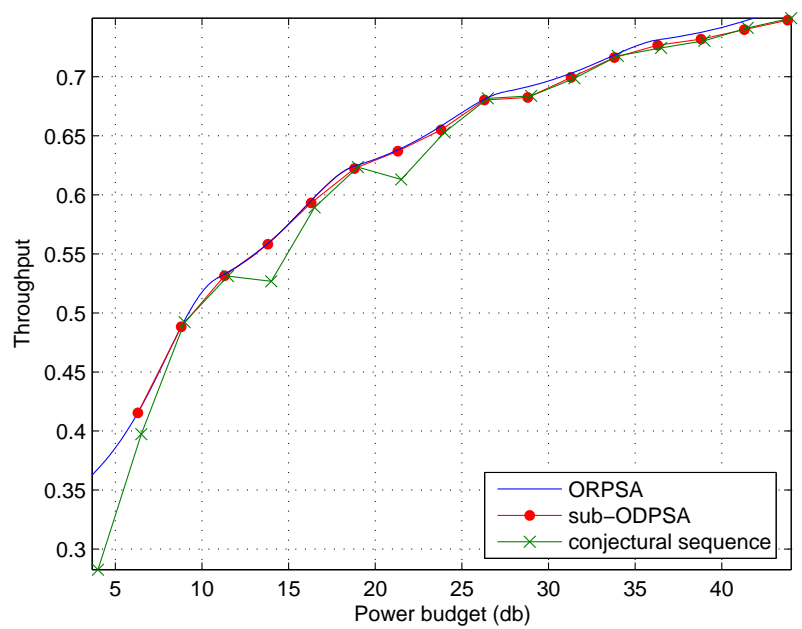

Fig. 13. Comparison of power-throughput characteristic of sub-ODPSA and conjectural sequence with the characteristic of ORPSA, which is shown to be an upper bound for the characteristic of ODPSA ( $D=9$ and $R=10$ ).

tions should be satisfied:

$$
\begin{gathered}
\frac{1-\tilde{\alpha}_{i}}{1-\tilde{\alpha}_{i+1}}=\exp \left(-\sum_{P^{\prime(j)}=P_{i}} \tilde{\gamma}_{j}\right), \quad 0 \leq i \leq L-1, \\
\sum_{i=0}^{L-1} R^{i}\left(\sum_{P^{\prime(j)}=P_{i}} \tilde{\gamma}_{j} / \tilde{\gamma}_{0}\right)-P_{a v}=0 .
\end{gathered}
$$

Assume that $i^{*}$ is the index of the power level used for state $D-1$, i.e. $P^{\prime(D-1)}=P_{i^{*}}$. Noting that this power level has been repeated for states $n \geq D$, the above equations will have the following form:

$$
\begin{aligned}
& \ln \left(\frac{1-\tilde{\alpha}_{i}}{1-\tilde{\alpha}_{i+1}}\right)+\sum_{P^{\prime(j)}=P_{i}}^{j<D} \tilde{\gamma}_{j}=0, \quad 0 \leq i \leq L-1, \quad i \neq i^{*} \\
& \ln \left(\frac{1-\tilde{\alpha}_{i^{*}}}{1-\tilde{\alpha}_{i^{*}+1}}\right)+\sum_{P^{\prime(j)}=P^{\prime(D-1)}}^{j<D} \tilde{\gamma}_{j}+\frac{\tilde{\gamma}_{D-1} \tilde{\alpha}_{i}^{*}}{1-\tilde{\alpha}_{i}^{*}}=0, \\
& \sum_{i=0}^{L-1} R^{i}\left(\sum_{P^{\prime(j)}=P_{i}}^{j<D} \tilde{\gamma}_{j} / \tilde{\gamma}_{0}\right)+\frac{R^{i^{\star}} \tilde{\gamma}_{D-1} \tilde{\alpha}_{i}^{*}}{\tilde{\gamma}_{0}\left(1-\tilde{\alpha}_{i}^{*}\right)}-P_{a v}=0 .
\end{aligned}
$$

After solving these equations for a given power budget constraint $P_{a v}$, the sub-optimum throughput value will be revealed and sub-optimum power-throughput characteristic can be presented. The resulting sub-optimum power-throughput characteristic with $R=10$ has been compared with the characteristic of ORPSA in Fig. 13. It shows that the performance of sub-ODPSA is very close to characteristic of ORPSA, which has been proven to be an upper bound for ODPSA characteristic. Therefore, we conclude that the presented subODPSAs can be used as close approximations to ODPSAs, both in limited and unlimited power scenarios. The powerthroughput characteristic of the conjectural sequence can be derived based on similar equations and has also been presented in Fig. 13.

\section{CONCLUSION}

In this paper, we discussed optimum random and deterministic power selection algorithms in conjunction with exponential backoff retransmission scheme in Aloha networks. For random algorithms, we first proved the inefficiency of fixed backoff factors and then by considering the constrained power budget scenario, optimum random power-throughput characteristics were derived based on perfect capture model. Finally, by presenting an approximate method we were able to extend the perfect model characteristics to SIR-based model sub-optimum power-throughput characteristics, based on which, sub-optimum power steps, number of power levels, PMFs and backoff factors were presented for given powerbudget constraints. Next, we introduced the possibility of using deterministic power selection algorithms. It was shown that optimum random algorithms outperform deterministic ones both in constrained and un-constrained power budget scenarios. However, by introducing a search method we were able to present sub-optimum deterministic algorithms and sub-optimum deterministic power-throughput characteristics, which were shown to have a performance very close to that of optimum random algorithms. The closeness of random and deterministic power-throughput characteristics, encourages use of deterministic power selection algorithms with which no random power generator block will be needed. Similar approaches, as those presented for random algorithms, can be used to adapt the deterministic perfect model results to SIRbased model.

\section{ACKNOWLEDGMENT}

The authors would like to thank Prof. F. Marvasti, director of Advanced Communications Research Center, Sharif University of Technology, Tehran, Iran, for his cooperation with this research. We are also thankful to Mr. V. Vahidiazar for his help with the simulation code.

\section{REFERENCES}

[1] J. J. Metzner, "On improving utilization in ALOHA network," IEEE Trans. Commun., vol. 24, pp. 447-448, Apr. 1976.

[2] C. C. Lee, "Random signal levels for channel access in packet broadcast networks," IEEE J. Select. Areas Commun., vol. 5, pp. 1026-1034, July 1987.

[3] R. O. LaMaire, A. Krishna, and M. Zorzi, "On the randomization of transmitter power levels to increase throughput in multiple access radio systems," Wireless Networks, vol. 4, pp. 263-277, Mar. 1998.

[4] Y. Leung, "Mean power consumption of artificial power capture in wireless networks," IEEE Trans. Commun., vol. 45, pp. 957-964, Aug. 1997.

[5] W. Luo and A. Ephremides, "Power levels and packet lengths in random multiple access," IEEE Trans. Inform. Theory, vol. 48, pp. 46-58, Jan. 2002.

[6] J. Luo and A. Ephremides, "Power levels and packet lengths in random multiple access with multiple-packet reception capability," IEEE Trans. Inform. Theory, vol. 52, pp. 414-420, Feb. 2006.

[7] B. Tsybakov, "Packet multiple access for channel with binary feedback, capture, and multiple reception," IEEE Trans. Inform. Theory, vol. 50, pp. 1073-1085, June 2004.

[8] M. Zorzi and R. R. Rao, "Capture and retransmission control in mobile radio," IEEE J. Select. Areas Commun., vol. 12, p. 12891298, Oct. 1994.

[9] J. Sanchez-Garcia and D. R. Smith, "Capture probability in rician fading channels with power control in the transmitters," IEEE Trans. Commun., vol. 50, pp. 1889-1891, Dec. 2002. 
[10] E. Altman, D. Barman, A. Benslimane, and R. E. Azouzi, "Slotted Aloha with priorities and random power," in Proc. Networking, Ontario, Canada, May 2005, pp. 610-622.

[11] B. Chu, "Improving IEEE 802.11 performance with power control and distance-based contention window selection," Master's thesis, University of Illinois at Urbana-Champaign, 2005.

[12] G. Bianchi, "Performance analysis of the IEEE 802.11 distributed coordination function," IEEE J. Select. Areas Commun., vol. 18, pp. 535-547, Mar. 2000.

[13] B. J. Kwak, N. O. Song, and L. Miller, "Performance analysis of Exponential Backoff," IEEE Trans. Wireless Commun., vol. 13, pp. 343355, Apr. 2005.

[14] Air Interface for Fixed Broadband Wireless Access Systems, IEEE Std. 802.16, 2001.

[15] B. Khoshnevis and B. H. Khalaj, "On a decentralized deterministic transmission power level selection algorithm in large Aloha networks under saturation," in Proc. IEEE International Conference on Communications (ICC'06), Istanbul, Turkey, June 2006, pp. 5732-5735.

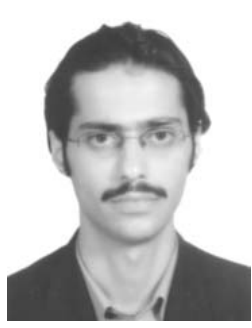

Behrouz Khoshnevis received B.Sc. and M.Sc. degrees from Sharif University of Technology, Tehran, Iran, in 2003 and 2005, respectively, both in Electrical Engineering. From 2003 to 2006, he was with the Advanced Communications Research Institute, Sharif University of Technology, Tehran, Iran. He is currently pursuing the Ph.D. degree in Electrical and Computer Engineering at the University of Toronto, Toronto, Ontario, Canada. Behrouz Khoshnevis is a member of IEEE Communications Society.

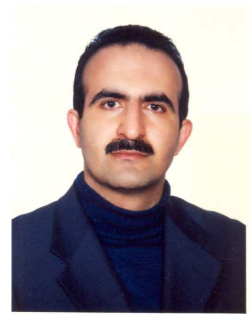

Babak H. Khalaj Babak H. Khalaj received the B.Sc. degree from Sharif University of Technology, Tehran, Iran, in 1989 and the M.Sc. and Ph.D. degrees from Stanford University, Stanford, CA, in 1992 and 1995, respectively, all in electrical engineering.

He joined KLA-Tencor in 1995 as a Senior Algorithm Designer, working on advanced processing techniques for signal estimation. From 1996 to 1999, he was with Advanced Fiber Communications and Ikanos Communications. Since then, he has been a Senior Consultant in the area of data communications. He was the co-editor of the Special Compatibility Standard Draft for ANSI T1E1 group from 1998 to 1999 , and he is the author of two U.S. patents and many papers in the signal processing and digital communications area. 